\title{
Fibroblast growth factors $I$ and 2 in cerebrospinal fluid are associated with HIV disease, methamphetamine use, and neurocognitive functioning
}

\author{
This article was published in the following Dove Press journal: \\ HIVIAIDS - Research and Palliative Care \\ 29 April 2016 \\ Number of times this article has been viewed
}

\section{Ajay R Bharti' \\ Steven Paul Woods ${ }^{2}$ \\ Ronald J Ellis ${ }^{3}$ \\ Mariana Cherner ${ }^{2}$ \\ Debra Rosario 3 \\ Michael Potter ${ }^{3}$ \\ Robert K Heaton ${ }^{2}$ \\ Ian P Everall ${ }^{4}$ \\ Eliezer Masliah ${ }^{5}$ \\ Igor Grant ${ }^{2}$ \\ Scott L Letendre'}

On behalf of the Translational Methamphetamine AIDS Research Center Group

'Department of Medicine, ${ }^{2}$ Department of Psychiatry,

${ }^{3}$ Department of Neurosciences, University of California San Diego, San Diego, CA, USA; ${ }^{4}$ Department of Psychiatry, University of Melbourne, Victoria, Australia; ${ }^{5}$ Department of Pathology, University of Californa San Diego, San Diego, CA, USA
Correspondence: Scott L Letendre Department of Medicine, University of California San Diego, 220 Dickinson Street, Suite A, San Diego, CA 92103, USA Tel +l 6195434730

Fax +I 6195435066

Email sletendre@ucsd.edu
Background: Human immunodeficiency virus (HIV) and methamphetamine use commonly affect neurocognitive (NC) functioning. We evaluated the relationships between NC functioning and two fibroblast growth factors (FGFs) in volunteers who differed in HIV serostatus and methamphetamine dependence (MAD).

Methods: A total of 100 volunteers were categorized into four groups based on HIV serostatus and MAD in the prior year. FGF-1 and FGF-2 were measured in cerebrospinal fluid by enzymelinked immunosorbent assays along with two reference biomarkers (monocyte chemotactic protein [MCP]-1 and neopterin). Comprehensive NC testing was summarized by global and domain impairment ratings.

Results: Sixty-three volunteers were HIV+ and 59 had a history of MAD. FGF-1, FGF-2, and both reference biomarkers differed by HIV and MAD status. For example, FGF-1 levels were lower in subjects who had either HIV or MAD than in HIV-and MAD- controls $(P=0.003)$. Multivariable regression identified that global NC impairment was associated with an interaction between FGF-1 and FGF-2 (model $\mathrm{R}^{2}=0.09, P=0.01$ ): higher FGF-2 levels were only associated with neurocognitive impairment among subjects who had lower FGF-1 levels. Including other covariates in the model (including antidepressant use) strengthened the model (model $\mathrm{R}^{2}=0.18, P=0.004$ ) but did not weaken the association with FGF-1 and FGF-2. Lower FGF-1 levels were associated with impairment in five of seven cognitive domains, more than FGF-2, MCP-1, or neopterin.

Conclusion: These findings provide in vivo support that HIV and MAD alter expression of FGFs, which may contribute to the NC abnormalities associated with these conditions. These cross-sectional findings cannot establish causality and the therapeutic benefits of recombinant FGF-1 need to be investigated.

Keywords: biomarker, cerebrospinal fluid, fibroblast growth factor, HIV, methamphetamine, HIV-associated neurocognitive disorders, HAND, neurocognitive impairment

\section{Background}

Human immunodeficiency virus (HIV) infects the central nervous system (CNS) and can cause HIV-associated neurocognitive disorders that range in severity from asymptomatic neurocognitive impairment (ANI) to HIV-associated dementia (HAD). ${ }^{1}$ HIV specifically infects perivascular macrophages, microglia, and, to a limited extent, astrocytes and this is followed by the release of proinflammatory cytokines and viral proteins that can lead to reversible and sometimes irreversible neuronal injury. ${ }^{2}$ These neuropathological changes can be exacerbated by the use of drugs of abuse, such as methamphetamine (MA). ${ }^{3}$ 
While immune and glial activation play influential roles in HIV pathogenesis in the CNS, dysregulation of neuroprotective mechanisms may also contribute.

Fibroblast growth factors (FGFs) are expressed in the brain and are involved in brain development and neuroprotection. The FGF family has diverse physiologic functions, including cell differentiation, migration, and survival. In the brain, FGF-1 is primarily produced by neurons, promotes neuronal survival, and may protect calbindin-immunoreactive interneurons from the neurotoxic effects of HIV-gp120.4-7 FGF-2 is produced by astrocytes as well as neurons and sustains endothelial cell fitness and blood-brain barrier (BBB) homeostasis. FGF-2 may also have neuroprotective properties, for example, it seems to protect against ischemic challenges. ${ }^{8-13}$ Of potential concern for HIV disease-related pathology, FGF-2 may upregulate the HIV co-receptor, CXCR4, in a dose-dependent manner. ${ }^{6}$ FGF-2 may also influence neural precursor cells. Of potential significance, FGF administration could be a new therapeutic modality for HIV-associated neurocognitive disorders: recombinant FGFs have been synthesized and are being evaluated in such diverse conditions as vascular disease, ${ }^{14}$ chronic kidney disease, ${ }^{15}$ and wound healing. ${ }^{16}$

Since cerebrospinal fluid (CSF) is in direct contact with the brain, concentrations of FGFs in CSF may reflect the expression of these proteins in neurons and glia. Few published studies have reported concentrations of FGFs in the CSF of humans. One early study reported FGF-like activity in the CSF of subjects who suffered from a traumatic brain injury. ${ }^{17}$ Two other studies identified that FGF-2 was elevated in the CSF of subjects with either amyotrophic lateral sclerosis (ALS) ${ }^{18}$ or moyamoya. ${ }^{19}$ A recent study showed a similar elevation of FGF-1 in the CSF of patients with Alzheimer's disease. ${ }^{20}$

The objective of this analysis was to evaluate the relationships between two FGFs and neurocognitive (NC) disease in subjects who differed by HIV and MA characteristics. To accomplish this, we assessed volunteers with a standardized neuromedical examination, lumbar puncture, and a comprehensive battery of NC tests. We also measured two reference biomarkers (monocyte chemotactic protein [MCP]-1 and neopterin) that have been implicated in HIV-associated NC disease. ${ }^{21,22}$

\section{Methods}

Participants were 100 volunteers who enrolled in a US National Institute on Drug Abuse-funded program (P01 DA12065) at University of California San Diego (UCSD)'s HIV Neurobehavioral Research Program (HNRP). Subjects were categorized into four groups based on the presence or absence of HIV disease and methamphetamine dependence (MAD) within the prior year. MAD was diagnosed using the Composite International Diagnostic Interview. In the parent project, subjects were excluded if they had used MA within 2 weeks or if they had a history of psychotic disorders, neurological disorders (eg, epilepsy), head injury, or loss of consciousness of $\geq 30$ minutes, or other substance use disorders (except cannabis). Volunteers were selected for this subanalysis if they were hepatitis $\mathrm{C}$ virus (HCV) seronegative, successfully underwent lumbar puncture, and had sufficient CSF in storage at $-80^{\circ} \mathrm{C}$ to perform the biomarker assays of interest. This study was reviewed by and received ethics approval from the UCSD Human Research Protections Program (http://irb.ucsd.edu/). All subjects provided written informed consent prior to enrollment.

Participants underwent a standardized comprehensive NC assessment. ${ }^{3}$ Seven cognitive abilities that may be affected by HIV and MA were tested: verbal fluency; attention and working memory; speed of information processing; learning; delayed recall; executive functions; and motor skills. A global deficit score (GDS) summarized participants' overall performance on the NC tests, raw performance on which was adjusted for age, education, sex, and race/ethnicity. ${ }^{3}$ This continuous metric ranged between 0 and 5 . A score of 0.5 is consistent with definite impairment, with higher scores indicating increasingly severe NC impairment.

HIV infection was diagnosed by standard clinical antibody detection. HIV RNA were measured in plasma and CSF using an assay that had a nominal detection limit of 50 copies $/ \mathrm{mL}$ (c/mL). CD4+ T-cell counts were measured in blood by routine clinical flow cytometry. Absence of recent recreational drug use was confirmed by testing the urine of each subject with an on-site screening test for common recreational drugs (including amphetamines, cocaine, barbiturates, cannabinoids, opiates, benzodiazepines, and phencyclidines). CSF was collected by routine lumbar puncture using a 22-gauge atraumatic needle.

FGF-1 and FGF-2 were measured by commercial enzyme-linked immunosorbent assays (ELISA) (R\&D Systems, Minneapolis, MN, USA). The lower limits of detection were $5.7 \mathrm{pg} / \mathrm{mL}$ for FGF-1 (DFB00b) and $3.0 \mathrm{pg} / \mathrm{mL}$ for FGF-2 (DFB50). MCP-1 was measured using a bead-based suspension immunoassay (Millipore, Billerica, MA, USA; lower limit of detection $3.2 \mathrm{pg} / \mathrm{mL}$ ). Neopterin was measured using a commercial ELISA (GenWay Biotech, San Diego, CA, USA; Cat 40-371-25012; limit of detection $0.7 \mathrm{nmol} / \mathrm{L}$ ). All assays were performed in duplicate. All coefficients of variation between duplicate wells were below $20 \%$. 


\section{Statistical analysis}

Data were analyzed using JMP software (SAS Institute, Cary, NC, USA). Values of some variables, including all four biomarkers, were transformed to improve the symmetry of their distribution. When assumptions were met, parametric tests (Student's $t$-tests, Pearson's correlation coefficient) were used. When assumptions were not met, nonparametric tests were used. Analysis of variance was used to analyze differences between more than two groups. Multivariable analyses were performed in two stages. In the first stage, stepwise linear regression was performed using HIV status, MAD group, FGF-1 and FGF-2 along with first-order interactions as candidate covariates. Second-stage modeling added demographic and disease characteristics (eg, HIV RNA levels) as well as levels of the two reference biomarkers (MCP-1 and neopterin) as candidate covariates. Antidepressant use was included as a candidate covariate in second-stage modeling since it has been linked to FGF expression. ${ }^{23,24}$ In both stages, the Akaike Information Criterion was used to determine relative goodness-of-fit. When interactions were identified, recursive partitioning was used to visualize the nature of the interaction using $10 \%$ of the sample as the minimum split size.

\section{Results \\ Subject characteristics}

Subjects were mostly middle-aged (mean 39.5 years), White (68\%) men (95\%) with some college education (mean 12.9 years). Sixty-three were HIV+ and 59 had a history of MAD. The four groups defined by HIV and MAD status differed in sex $(P=0.04)$ and antidepressant use $(P=0.002)$ and tended to differ in education $(P=0.08)$ but did not differ in other demographic characteristics or by global NC impairment. By design, all subjects were HCV seronegative. The two HIV+ groups did not differ in their HIV disease characteristics, except that more MAD+ subjects were taking failing antiretroviral therapy (ART) (ie, they were taking ART but had HIV RNA levels $>50 \mathrm{c} / \mathrm{mL}$ ). Subject characteristics are summarized in Table 1.

\section{FGFs, MCP-I, and neopterin}

FGF-1 in CSF ranged from 5.7 to $1,532 \mathrm{pg} / \mathrm{mL}$ (median 40.2, interquartile range [IQR] 26.3-83.3). FGF-2 in CSF ranged from 3.2 to $43.5 \mathrm{pg} / \mathrm{mL}$ (median 7.7, IQR 6-12.4). MCP-1 in CSF ranged from 216.6 to $2,557 \mathrm{pg} / \mathrm{mL}$ (median 507.8, IQR 417.7-693.2) and neopterin in CSF ranged from 2.4 to $59.7 \mathrm{nmol} / \mathrm{mL}$ (median 7.5, IQR 5.4-12.2).

FGF-1 and FGF-2 correlated with each other $(\rho=-0.41$, $P<0.0001$ ). Each correlated with MCP-1 (FGF-1: $\rho=0.33$,
$P=0.0009$; FGF-2: $\rho=-0.54, P<0.0001)$ but neither strongly correlated with neopterin (FGF-1: $\rho=0.04, P=0.68$; FGF-2: $\rho=-0.16, P=0.12$ ).

All four biomarkers differed by HIV or MAD status (Table 1) with differing patterns of association. FGF-1 was much higher in control subjects $(\mathrm{d}=1.2, P=0.02$ for the comparison between HIV-MAD- vs all others) but did not differ between the three other groups $(P=0.67)$. FGF- 2 was higher in subjects who had a history of MAD $(\mathrm{d}=0.80, P<0.0001)$ but did not differ by HIV status ( $P=0.34)$. MCP-1 was lower in the HIV-MAD+ group $(\mathrm{d}=0.80, P=0.0002)$ but did not differ among the other 3 groups $(P=0.29)$. HIV+ subjects had higher neopterin levels $(\mathrm{d}=0.70, P=0.0004)$ but MAD+ subjects trended toward lower levels $(\mathrm{d}=0.37, P=0.09)$. Since the four HIV/MAD groups differed by sex, antidepressant use, and education (Table 1), we adjusted for these covariates in multivariable linear regression models predicting each biomarker. The observed associations between HIV, MAD, and each biomarker did not significantly change after these adjustments.

Among HIV+ individuals, several biomarkers tended to differ based on plasma HIV RNA suppression but none of the comparisons reached statistical significance (mean MCP-1 $567.1 \mathrm{pg} / \mathrm{mL}$ when plasma HIV RNA undetectable vs $713.8 \mathrm{pg} / \mathrm{mL}$ when detectable, $P=0.12$; mean neopterin $9.7 \mathrm{nmol} / \mathrm{mL}$ when plasma HIV RNA undetectable vs 12.7 when detectable, $P=0.17$; mean FGF-2 $11.2 \mathrm{pg} / \mathrm{mL}$ when plasma HIV RNA undetectable vs 8.8 when detectable, $P=0.16)$. FGF-1 in CSF was not associated with either HIV RNA levels or ART use. None of the biomarkers correlated with current or nadir CD4+ T-cell counts.

\section{Associations with global NC impairment}

In this subgroup ( $\mathrm{n}=100)$ of the larger cohor $^{25}$ that met the selection criteria, global neurocognitive impairment (NCI) was not statistically significantly associated with either HIV seropositivity (relative risk $1.25, P=0.23$ ) or history of MAD (relative risk 1.13, $P=0.65$ ). Global NCI was also not associated with demographic characteristics, although it trended toward an association with White ethnicity (77.8\% of impaired subjects were White vs $59.7 \%$ of unimpaired subjects, $P=0.11$ ).

Global NCI was associated with lower FGF-1 levels in CSF ( $\mathrm{d}=0.47, P=0.003)$ but not with levels of FGF-2 ( $P=0.38)$, MCP-1 $(P=0.22)$, or neopterin $(P=0.41)$. Firststage multivariable modeling identified that global NCI was associated with an interaction between FGF-1 and FGF-2 (model $\mathrm{R}^{2}=0.09, P=0.01$ ). Recursive partitioning identified 
Table I Demographic and disease characteristics of research subjects, stratified by neurocognitive impairment status

\begin{tabular}{|c|c|c|c|c|c|}
\hline & \multicolumn{5}{|l|}{ Groups } \\
\hline & HIV-MAD- $(\mathrm{N}=12)$ & HIV-MAD+ (N=25) & HIV+MAD- $(\mathbf{N}=29)$ & HIV+MAD+ (N=34) & $P$-value \\
\hline Age (years, mean $\pm S D)$ & $42.3 \pm 9.4$ & $38.7 \pm 9.4$ & $39.1 \pm 8.6$ & $37.1 \pm 7.3$ & 0.33 \\
\hline Sex (women, \%) & 25.0 & 0.0 & 3.4 & 2.9 & 0.04 \\
\hline Ethnicity (White, \%) & 50.0 & 60.0 & 71.4 & 67.6 & 0.57 \\
\hline Education (years, mean $\pm S D$ ) & $12.1 \pm 2.1$ & $12.4 \pm 2.0$ & $12.9 \pm 1.4$ & $13.5 \pm 2.1$ & 0.08 \\
\hline HCV serostatus (positive, \%) & 0.0 & 0.0 & 0.0 & 0.0 & - \\
\hline Global neurocognitive impairment (\%) & 16.7 & 27.6 & 44.0 & 20.6 & 0.19 \\
\hline Antidepressant use (\%) & 0.0 & 20.0 & 41.4 & 47.1 & 0.002 \\
\hline FGF-I, CSF (mean $\pm S D, p g / m L)$ & $418.0 \pm 497$ & $47.1 \pm 35.5$ & $91.6 \pm 131.8$ & $92.4 \pm|5| .4$ & 0.003 \\
\hline FGF-2, CSF (mean \pm SD, pg/mL) & $6.8 \pm 1.2$ & $12.2 \pm 5.5$ & $7.8 \pm 3.2$ & $11.2 \pm 7.5$ & 0.001 \\
\hline MCP-I, CSF (mean \pm SD, pg/mL) & $706.2 \pm 347.0$ & $443.2 \pm 142.7$ & $700.9 \pm 307.7$ & $621.3 \pm 421.9$ & 0.002 \\
\hline Neopterin, CSF (mean $\pm \mathrm{SD}, \mathrm{pg} / \mathrm{mL})$ & $8.1 \pm 6.6$ & $6.6 \pm 2.9$ & $13.9 \pm 12.5$ & $10.3 \pm 7.2$ & 0.005 \\
\hline Current CD4+ T-cell count (mean $\pm \mathrm{SD}, \mu \mathrm{L}^{-1}$ ) & NA & NA & $530 \pm 269$ & $48 I \pm 279$ & 0.48 \\
\hline Nadir CD4+ T-cell count $\left(\right.$ mean $\left.\pm S D, / \mu \mathrm{L}^{-1}\right)$ & NA & NA & $263 \pm 225$ & $311 \pm 216$ & 0.39 \\
\hline AIDS (with diagnosis, \%) & NA & NA & 51.7 & 32.4 & 0.12 \\
\hline Current ART use (\%) & NA & NA & 65.5 & 44.1 & 0.13 \\
\hline \multicolumn{6}{|l|}{ Among those taking ART } \\
\hline $\mathrm{CPE}($ mean $\pm \mathrm{SD})$ & NA & NA & $7.3 \pm 2.5$ & $7.7 \pm 2.2$ & 0.67 \\
\hline HIV RNA, plasma $(\%<50 \mathrm{c} / \mathrm{mL})$ & NA & NA & 73.7 & 26.7 & 0.01 \\
\hline HIV RNA, CSF $(\%<50 \mathrm{c} / \mathrm{mL}, \mathrm{n}=60)$ & NA & NA & 94.4 & 57.1 & 0.03 \\
\hline
\end{tabular}

Abbreviations: AIDS, acquired immune deficiency syndrome; ART, antiretroviral therapy; CPE, CNS penetration score; CSF, cerebrospinal fluid; FGF, fibroblast growth factor; HCV, hepatitis C virus; HIV, human immunodeficiency virus; MAD, methamphetamine dependence; MCP, monocyte chemotactic protein; NA, not applicable; RNA, ribonucleic acid; SD, standard deviation.

the nature of the interaction: higher FGF-2 levels were only associated with NCI among those with lower FGF-1 levels (Figure 1A). The second-stage modeling identified additional covariates that strengthened the model but the interaction between FGF-1 and FGF-2 was not weakened by their inclusion (model $\mathrm{R}^{2}=0.18, P=0.004$ ). Covariates that explained additional variance in global NCI included HIV serostatus (parameter estimate $P=0.03$ ) and an interaction between FGF-1 and neopterin (parameter estimate $P=0.02$ ). Recursive partitioning identified that the nature of the interaction was similar to the one between FGF-1 and FGF-2: higher neopterin levels were associated with NCI only among those with lower FGF-1 levels (Figure 1B).

Among people living with HIV disease, global NCI trended toward an association with lower FGF-1 levels ( $P=0.109$ ) but not with demographic, disease, or treatment characteristics. The size of the HIV+ sample $(n=63)$ limited multivariable models. In first-stage models, only the interaction between FGF-1 and FGF-2 entered the model of global $\mathrm{NCI}\left(\mathrm{R}^{2}=0.09, P=0.01\right)$. In the second-stage of modeling that accounted for HIV and ART characteristics, no additional covariates entered the model.

Among the 37 subjects who had undetectable HIV RNA levels in plasma, global NCI was associated with older age (mean 43.3 years vs 38.7 years, $P=0.03$ ) and trended toward associations with lower FGF-1 levels $(P=0.106)$ and lower CNS penetration score values (mean 5.7 vs 8.5 , $\mathrm{d}=1.0, P=0.06$ ) but not with other demographic, disease, or treatment characteristics. Considering the small size of the subgroup with suppressed plasma HIV RNA in this analysis, no multivariable modeling was performed.

\section{Associations with domain $\mathrm{NCl}$}

The patterns of associations of the two FGFs and the two reference biomarkers with impairment in specific cognitive domains differed. Lower levels of FGF-1 at least trended toward association with impairment in the largest number of domains: learning $(\mathrm{d}=0.37, P=0.04)$, working memory $(\mathrm{d}=0.36, P=0.07)$, recall ( $\mathrm{d}=0.33, P=0.10)$, speed of information processing ( $\mathrm{d}=0.40, P=0.12$ ), and motor functioning ( $\mathrm{d}=0.34, P=0.12$ ). Higher FGF-2 levels trended toward association with speed of information processing impairment $(\mathrm{d}=0.69, P=0.11)$ while lower levels were associated with verbal functioning impairment $(\mathrm{d}=0.40, P=0.05)$. Higher MCP-1 levels were associated with motor impairment $(\mathrm{d}=0.62, P=0.03)$ while lower levels were associated with speed of information processing impairment ( $\mathrm{d}=0.84, P=0.007)$. Neopterin was not associated with impairment in any single domain. The domain that had the strongest association for neopterin was motor functioning: the association between motor impairment and neopterin had a small effect size $(\mathrm{d}=0.30, P=0.19)$.

The domains most commonly correlated with these four biomarkers were motor functioning and speed of information processing. Multivariable analysis identified that motor 
A

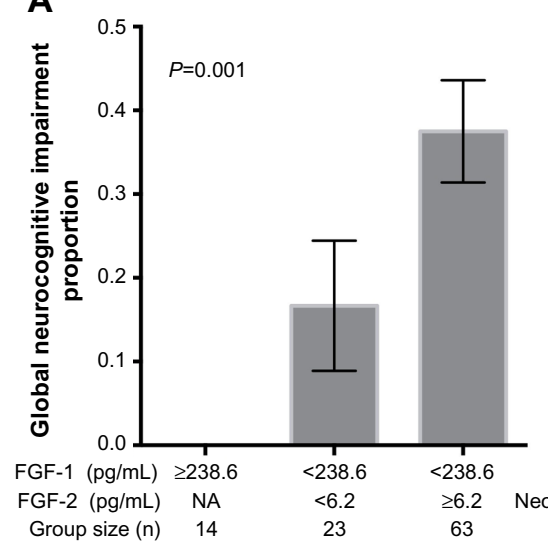

B

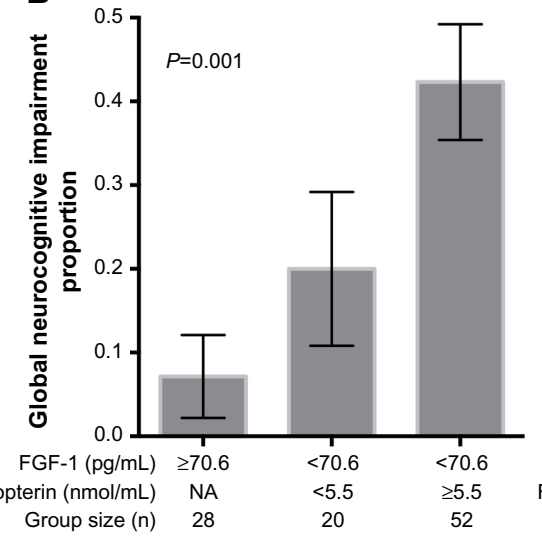

C

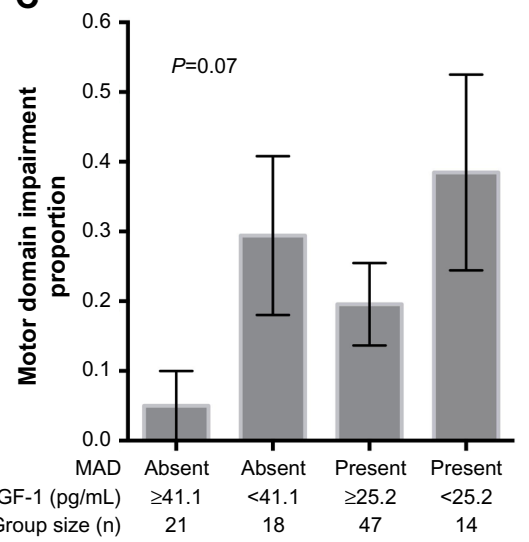

Figure I Interactions between FGF-I and neurocognitive functioning.

Notes: The relationship between FGF-I and global NCl was modified by either FGF-2 (A) or neopterin (B). (C) The relationship between FGF-I and motor impairment was modified by MAD. The threshold values of FGF-I differ between figures because they derive from different recursive partitioning models.

Abbreviations: FGF, fibroblast growth factors; MAD, methamphetamine dependence; NA, not applicable; $\mathrm{NCl}$, neurocognitive impairment.

impairment was associated with HIV disease, MAD, and levels of three of the four biomarkers (FGF-1, FGF-2, and MCP-1) (model $\mathrm{R}^{2}=0.41, P<0.0001$ ). This model included an interaction between MAD and FGF-1 that was visualized using recursive partitioning (Figure 1C). Speed of information processing impairment was associated only with lower MCP-1 levels (model $\mathrm{R}^{2}=0.10, P=0.03$ ), although modeling was limited by the small number of subjects with speed of information processing impairment $(n=6)$.

\section{Discussion}

Our analysis is the largest to date to measure FGFs in CSF from adults living with HIV disease and the first to compare them to MAD. A smaller published report identified lower FGF-2 concentrations in CSF collected from 19 individuals with HIV-associated minor NC disorder compared with HIV seronegative controls. ${ }^{26}$ Our primary findings were that 1) lower FGF-1 concentrations in CSF were associated with HIV disease, MAD, and NCI and 2) higher concentrations of FGF-2 were associated with MAD (but not with HIV) and influenced the relationship between FGF-1 and NC functioning.

These findings provide in vivo support for the neuroprotective properties of FGF-1. Such a role was previously proposed by investigators in our group based on the observation that neurodegeneration was associated with a reduction in the FGF-1 expression in brain tissue from individuals with antemortem NCI and postmortem HIV encephalitis. ${ }^{4}$ Our group also showed that FGF-1 was protective against neurotoxicity due to the HIV envelope protein gp120 in primary human neuronal cultures. ${ }^{4}$ Using transgenic mice expressing human FGF-1, we also demonstrated a similar benefit that might be due to inactivation of glycogen synthase kinase (GSK) $3 \beta .27$

In contrast, FGF-2 appears to be predominantly associated with neurologic injury since higher FGF-2 concentrations in CSF were associated with global NCI. The possible deleterious effects of FGF-2 are consistent with reports that FGF-2 mRNA levels are elevated in the frontal cortex of cognitively impaired acquired immune deficiency syndrome (AIDS) patients ${ }^{28}$ and that FGF-2 may contribute to neuronal damage in multiple sclerosis. ${ }^{29}$ An alternative explanation for our findings is that the reported neuroprotective effects of FGF-2 $2^{30,31}$ are either inadequate in the presence of chronic disease or are influenced by other factors, which is supported by the observed interaction between FGF-2 and FGF-1. The strong correlation between FGF-2 and MCP-1 raises concerns about colinearity in multivariable analyses. Longitudinal data, particularly following a therapeutic intervention, might better discern the distinct effects of FGF-2.

Analyses of individual cognitive domains also support that FGF-1 and FGF-2 may lead to injury of different neural circuits. Multiple biomarkers, including FGF-1, were associated with motor functioning and speed of information processing but, among our small panel of biomarkers, only FGF-1 was associated with learning and memory impairment. Dopaminergic systems subserve these functions and can be adversely affected by either HIV or MAD. While other circuits support learning and memory, support for the relationship between FGF-1 and dopaminergic systems is present for the subjects included in this analysis: lower FGF-1 levels correlated with higher levels of homovanillic acid, a catecholamine metabolite, in CSF $(\mathrm{r}=-0.23, P=0.04)$ and higher dopamine-to-homovanillic acid ratios $(\mathrm{r}=-0.27$, 
$P=0.01$; data not shown). The domain analysis also identified that FGF-2 and MCP-1 may be associated with cognitive abilities differently; higher levels were associated with impairment in one domain while lower levels were associated with impairment in another. While these findings could be due to type I statistical error, they could also explain why the strength of associations with MCP-1, for instance, has varied from study to study (ie, the findings depend on the prevalence of impaired domains in each cohort).

An important limitation of our project is that we only measured FGFs in CSF, not in blood. While CSF likely provides a better window into CNS events than blood, we could have more confidently ascribed the production of FGFs to glia and neurons if we had shown the absence of significant associations with FGF concentrations in blood. Instead, we must consider the possibility that our observations are due to events outside the CNS since blood-CSF barrier and BBB permeability perturbations might have allowed diffusion of blood-derived FGFs into CSF. This might be particularly true since MA can alter BBB permeability by modulating tight junction expression to decrease transendothelial resistance as well as enhanced transendothelial migration of immune cells. ${ }^{32}$ Another important limitation is the cross-sectional design of our analyses. Such designs are inherently limited in their ability to account for bias, particularly bias due to inter-individual differences in time-linked factors such as HIV disease trajectory or the density of MA use over time. Cross-sectional designs are also very limited in their ability to determine causality. This analysis also included relatively few HIV+ subjects who were taking suppressive antiretroviral therapy. Thus, while the $2 \times 2$ design enables inferences about the associations between HIV, MAD, and FGFs, the findings may not generalize well to effectively treated $\mathrm{HIV}+$ patients in the clinic.

These findings may be particularly important since human recombinant FGF-1 is being evaluated as a clinical treatment, albeit for other indications (coronary artery disease [NCT00117936] and peripheral vascular disease [NCT00424866]). Even if a future clinical trial demonstrates that human recombinant FGF-1 safely and effectively treats HIV- or MA-associated NCI, its parenteral administration will limit its use. Orally administered drugs would be more clinically useful. If our findings are due, in part, to modulation of downstream molecules such as GSK3 $\beta$, then modulators of signaling pathways, such as oral lithium, might also protect the CNS from HIV-related neurotoxicity. Consistent with this, lithium has evidence of in vitro and in vivo benefits in HIV disease. ${ }^{27}$
In conclusion, we have shown that HIV and MAD are each associated with reductions in FGF-1 levels in CSF and these reductions were associated with worse NC functioning. Further studies are required to confirm these findings and to determine if these associations are also present in effectively treated people living with HIV disease.

\section{Acknowledgments}

The research described in this paper was funded by the National Institutes on Drug Abuse (P01 DA12065 to IG). The National Institute of Mental Health provided salary support for Dr Bharti and Dr Letendre (K23 MH085512 to ARB, K24 MH097673 to SLL). An earlier version of this work was presented at the 9th International Symposium on Neurovirology, June 2009, Miami Beach, FL, USA.

\section{Disclosure}

The authors report no conflicts of interest in this work.

\section{References}

1. Antinori A, Arendt G, Becker JT, et al. Updated research nosology for HIV-associated neurocognitive disorders. Neurology. 2007;69(18):1789-1799.

2. McArthur JC, Steiner J, Sacktor N, Nath A. Human immunodeficiency virus-associated neurocognitive disorders: Mind the gap. Ann Neurol. 2010;67(6):699-714.

3. Letendre SL, Cherner M, Ellis RJ, et al. The effects of hepatitis C, HIV, and methamphetamine dependence on neuropsychological performance: biological correlates of disease. AIDS. 2005;19 Suppl 3: S72-S78.

4. Everall IP, Trillo-Pazos G, Bell C, Mallory M, Sanders V, Masliah E. Amelioration of neurotoxic effects of HIV envelope protein gp 120 by fibroblast growth factor: a strategy for neuroprotection. J Neuropathol Exp Neurol. 2001;60(3):293-301.

5. Mattson MP, Rychlik B, Chu C, Christakos S. Evidence for calciumreducing and excito-protective roles for the calcium-binding protein calbindin-D28k in cultured hippocampal neurons. Neuron. 1991;6(1):41-51.

6. Sanders VJ, Everall IP, Johnson RW, Masliah E. Fibroblast growth factor modulates HIV coreceptor CXCR4 expression by neural cells. HNRC Group. J Neurosci Res. 2000;59(5):671-679.

7. Thorns V, Licastro F, Masliah E. Locally reduced levels of acidic FGF lead to decreased expression of 28-kda calbindin and contribute to the selective vulnerability of the neurons in the entorhinal cortex in Alzheimer's disease. Neuropathology. 2001;21(3):203-211.

8. Dono R. Fibroblast growth factors as regulators of central nervous system development and function. Am J Physiol Regul Integr Comp Physiol. 2003;284(4):R867-R881.

9. Fulgham DL, Widhalm SR, Martin S, Coffin JD. FGF-2 dependent angiogenesis is a latent phenotype in basic fibroblast growth factor transgenic mice. Endothelium. 1999;6(3):185-195.

10. Klint P, Claesson-Welsh L. Signal transduction by fibroblast growth factor receptors. Front Biosci. 1999;4:D165-D177.

11. MacMillan V, Judge D, Wiseman A, Settles D, Swain J, Davis J. Mice expressing a bovine basic fibroblast growth factor transgene in the brain show increased resistance to hypoxemic-ischemic cerebral damage. Stroke. 1993;24(11):1735-1739.

12. Powers CJ, McLeskey SW, Wellstein A. Fibroblast growth factors, their receptors and signaling. Endocr Relat Cancer. 2000;7(3):165-197. 
13. Vaccarino FM, Schwartz ML, Raballo R, et al. Changes in cerebral cortex size are governed by fibroblast growth factor during embryogenesis. Nat Neurosci. 1999;2(3):246-253.

14. Berger JS, Hiatt WR. Medical therapy in peripheral artery disease. Circulation. 2012;126(4):491-500.

15. Lederman RJ, Mendelsohn FO, Anderson RD, et al. Therapeutic angiogenesis with recombinant fibroblast growth factor- 2 for intermittent claudication (the TRAFFIC study): a randomised trial. Lancet. 2002;359(9323):2053-2058.

16. Plichta JK, Radek KA. Sugar-coating wound repair: a review of FGF-10 and dermatan sulfate in wound healing and their potential application in burn wounds. J Burn Care Res. 2012;33(3):299-310.

17. Patterson SL, Grady MS, Bothwell M. Nerve growth factor and a fibroblast growth factor-like neurotrophic activity in cerebrospinal fluid of brain injured human patients. Brain Res. 1993;605(1):43-49.

18. Johansson A, Larsson A, Nygren I, Blennow K, Askmark H. Increased serum and cerebrospinal fluid FGF-2 levels in amyotrophic lateral sclerosis. Neuroreport. 2003;14(14):1867-1869.

19. Yoshimoto T, Houkin K, Takahashi A, Abe H. Evaluation of cytokines in cerebrospinal fluid from patients with moyamoya disease. Clin Neurol Neurosurg. 1997;99 Suppl 2:S218-S220.

20. Mashayekhi F, Hadavi M, Vaziri HR, Naji M. Increased acidic fibroblast growth factor concentrations in the serum and cerebrospinal fluid of patients with Alzheimer's disease. J Clin Neurosci. 2010;17(3):357-359.

21. Hagberg L, Fuchs D, Rosengren L, Gisslen M. Intrathecal immune activation is associated with cerebrospinal fluid markers of neuronal destruction in AIDS patients. $J$ Neuroimmunol. 2000;102(1):51-55.

22. Kelder W, McArthur JC, Nance-Sproson T, McClernon D, Griffin DE. Beta-chemokines MCP-1 and RANTES are selectively increased in cerebrospinal fluid of patients with human immunodeficiency virusassociated dementia. Ann Neurol. 1998;44(5):831-835.

23. He S, Zhang T, Hong B, et al. Decreased serum fibroblast growth factor-2 levels in pre- and post-treatment patients with major depressive disorder. Neurosci Lett. 2014;579:168-172.
24. Kajitani N, Hisaoka-Nakashima K, Morioka N, et al. Antidepressant acts on astrocytes leading to an increase in the expression of neurotrophic/ growth factors: differential regulation of FGF-2 by noradrenaline. PLoS One. 2012;7(12):e51197.

25. Carey CL, Woods SP, Rippeth JD, Gonzalez R, Heaton RK, Grant I. Additive deleterious effects of methamphetamine dependence and immunosuppression on neuropsychological functioning in HIV infection. AIDS Behav. 2006;10(2):185-190.

26. Albrecht D, Garcia L, Cartier L, et al. Trophic factors in cerebrospinal fluid and spinal cord of patients with tropical spastic paraparesis, HIV, and Creutzfeldt-Jakob disease. AIDS Res Hum Retroviruses. 2006;22(3):248-254.

27. Crews L, Patrick C, Achim CL, Everall IP, Masliah E. Molecular pathology of neuro-AIDS (CNS-HIV). Int J Mol Sci. 2009;10(3):1045-1063.

28. Boven LA, Middel J, Portegies P, Verhoef J, Jansen GH, Nottet HS. Overexpression of nerve growth factor and basic fibroblast growth factor in AIDS dementia complex. J Neuroimmunol. 1999;97(1-2):154-162.

29. Sarchielli P, Di Filippo M, Ercolani MV, et al. Fibroblast growth factor-2 levels are elevated in the cerebrospinal fluid of multiple sclerosis patients. Neurosci Lett. 2008;435(3):223-228.

30. Schindowski K, Belarbi K, Buee L. Neurotrophic factors in Alzheimer's disease: role of axonal transport. Genes Brain Behav. 2008;7 Suppl 1 : 43-56.

31. Rottlaender A, Villwock H, Addicks K, Kuerten S. Neuroprotective role of fibroblast growth factor- 2 in experimental autoimmune encephalomyelitis. Immunology. 2011;133(3):370-378.

32. Mahajan SD, Aalinkeel R, Sykes DE, et al. Methamphetamine alters blood brain barrier permeability via the modulation of tight junction expression: Implication for HIV-1 neuropathogenesis in the context of drug abuse. Brain Res. 2008;1203:133-148.
HIV/AIDS - Research and Palliative Care

\section{Publish your work in this journal}

HIV/AIDS - Research and Palliative Care is an international, peerreviewed open-access journal focusing on advances in research in HIV, its clinical progression and management options including antivira treatment, palliative care and public healthcare policies to control viral spread. The journal welcomes original research, basic science,

\section{Dovepress}

clinical \& epidemiological studies, reviews \& evaluations, expert opinion \& commentary, case reports \& extended reports. The manuscript management system is completely online and includes a very quick and fair peer-review system. Visit http://www.dovepress.com/ testimonials.php to read real quotes from published authors. 